\title{
Entropy and Selection: Life as an Adaptation for Universe Replication
}

\author{
Michael E. Price \\ Department of Life Sciences, Brunel University, London, UK \\ Correspondence should be addressed to Michael E. Price; michael.price@brunel.ac.uk
}

Received 21 February 2017; Accepted 28 May 2017; Published 20 June 2017

Academic Editor: Carlos Gershenson

Copyright (C) 2017 Michael E. Price. This is an open access article distributed under the Creative Commons Attribution License, which permits unrestricted use, distribution, and reproduction in any medium, provided the original work is properly cited.

\begin{abstract}
Natural selection is the strongest known antientropic process in the universe when operating at the biological level and may also operate at the cosmological level. Consideration of how biological natural selection creates adaptations may illuminate the consequences and significance of cosmological natural selection. An organismal trait is more likely to constitute an adaptation if characterized by more improbable complex order, and such order is the hallmark of biological selection. If the same is true of traits created by selection in general, then the more improbably ordered something is (i.e., the lower its entropy), the more likely it is to be a biological or cosmological adaptation. By this logic, intelligent life (as the least-entropic known entity) is more likely than black holes or anything else to be an adaptation designed by cosmological natural selection. This view contrasts with Smolin's suggestion that black holes are an adaptation designed by cosmological natural selection and that life is the by-product of selection for black holes. Selection may be the main or only ultimate antientropic process in the universe/multiverse; that is, much or all observed order may ultimately be the product or by-product of biological and cosmological selection.
\end{abstract}

\begin{abstract}
"Another, related meaning of entropy is that it is a measure of disorganization. The atoms in a gas are disordered to the extent that there is no way to tell one from another. In equilibrium there is maximal disorder, because every atom moves randomly, with the same average energy as any other atom. A living system, on the contrary, continually creates an enormous number of different kind of molecules, each of which generally perform a unique function. The entropy of a living thing is consequently much lower, atom for atom, than anything else in the world."

Smolin (1997), The Life of the Cosmos, p. 28.
\end{abstract}

\section{Introduction}

The second law of thermodynamics explains the everincreasing presence of disorder in physical systems. But this process of increasing entropy (the "entropic process") could not occur without some order in the first place. Is there also a fundamental process that accounts for the presence of order in physical systems? Such a process has certainly been identified for biological systems: natural selection causes the extraordinarily orderly (i.e., adaptive) properties of organisms. However, to what extent could the selective process, conceived in cosmological and not just biological terms, also account for most or even all order in the universe?

To appreciate the possible significance of selection as a general antientropic process, it helps to consider what we already know about how selection operates at the biological level. Such consideration suggests not only that selection may be the fundamental source of order in the universe-essentially, the opposite of the entropic process-but also that intelligent life is more likely than any other known thing in the universe to be an adaptation designed by cosmological natural selection. 


\section{Adaptations Designed by Biological Natural Selection}

Living organisms are the least entropic (i.e., the most complexly ordered and improbable) entities known to exist $[1,2]$. Biological natural selection (BNS) (intended as shorthand for all types of biological selection, including natural selection proper [3], sexual selection [4], and kin selection [5]), then, is the strongest known antientropic process, because it creates organisms. Organisms have extraordinarily low entropy because BNS endows them with adaptations that ultimately enable genetic replication. Achieving replication is a complex and highly ordered process and requires organisms that are themselves complex and highly ordered. That is, it requires organisms composed of myriad adaptations that are functionally specialized for solving diverse kinds of adaptive problems (such as breathing, seeing, digesting, and mating). By enabling the organism to succeed in the competition with conspecifics to survive and reproduce, the genes which encode these adaptations are ultimately able to replicate [5-7]. Genes are the replicators in this process, and the organism-which is essentially a bundle of adaptations-is their vehicle of selection [8]. In summary, then, the selective process works by favouring those genes which, by encoding adaptive designs that outcompete other designs, most successfully enable their own replication.

BNS is the only known process that can design an organismal adaptation, but not all organismal traits are adaptations. Traits may also be by-products of adaptations or just random noise that is irrelevant to replication and therefore invisible to selection. For example, an umbilical cord is an adaptation, but the navel it leaves behind is its by-product, and the precise size and shape of this navel are just noise. To assess whether an organismal trait is an adaptation or not, we look for evidence of special design [6]: how precisely designed is that adaptation for solving a specific adaptive problem, like a key that has been precisely engineered to fit a particular lock? To identify a trait as an adaptation is ultimately to make a probability assessment: what is the likelihood that this trait would have arisen by chance, as a narrowly tailored solution to a specific adaptive problem [7]? The more improbable a trait is in this regard-that is, the lower its entropy-the more likely it is to be an adaptation, as opposed to a by-product or noise.

For example, the mammalian eye is composed of dozens of diverse components that, through coordinated action, can convert electromagnetic radiation into information that is useful to the organism. We can be virtually 100 per cent certain that the eye is an adaptation, because it would be essentially impossible for such a functionally complex device to arise by chance. This level of certainty must be reduced when considering many other traits; however, even those which still seem very likely to be adaptations. For example, people whose relatively recent evolutionary ancestors lived for many generations in colder northern environments have less melanin in their skin than those whose ancestors hailed from sunnier equatorial regions. Their lighter skin is probably an adaptation designed to allow more ultraviolet radiation to penetrate the skin and thus allow the body to produce more vitamin D. Compared to the eye, however, reduced epidermal melanin is much less improbably complex and therefore much more likely to arise by chance (i.e., more entropic). Even if we are quite confident that reduced melanin is an adaptation to northern climates, its status as an adaptation is necessarily less certain than that of the eye.

\section{Adaptations Designed by Cosmological Natural Selection}

According to the theory of cosmological natural selection (CNS), we live in a multiverse in which the most-represented universe designs are those best at replication. Compared to BNS, CNS is a vastly less thoroughly documented and wellunderstood process. We can still reasonably consider, however, what the consequences would be if CNS were true. My goal here is not to present an extensive case for why CNS could plausibly occur, but rather to take an adaptationist perspective on the CNS theory that has already been developed by Smolin [1,9] and others [10]. According to this theory, black holes are the mechanism by which universes replicate, and universe designs are selected to produce greater numbers of black holes. However, if we apply the adaptationist logic outlined above to Smolin's model, then life appears more likely than black holes to be a mechanism of universe replication.

As noted above, we can judge the extent to which an organismal trait is likely to be an adaptation by conducting a probability assessment: traits that are more complexly ordered are less likely to arise by chance and thus more likely to be the direct product of the strongest documented antientropic process, BNS. Conducting this assessment is often relatively straightforward when the organism is the vehicle of selection, but what about when the universe is the vehicle? According to Smolin's model, black holes are adaptations designed by CNS. Other theorists, however, have proposed that intelligent life may constitute such an adaptation, an idea known as "cosmological natural selection with intelligence" or CNS-I [11] (or alternatively, "cosmological artificial selection" [12]). CNS-I models propose that intelligence functions ultimately (after evolving to a sufficiently sophisticated state) to create new universes that replicate the physical laws and parameters of its home universe [11-15].

So which is more likely to be an adaptation for the replication of universes, intelligent life or black holes? Based on adaptationist logic, life would seem to be the more promising candidate of the two, simply because it is far more complexly ordered and less likely to arise by chance. As the most improbable known thing in the universe, designed by the strongest known antientropic process, life seems more likely than black holes-or any other known entity-to be a CNS-designed adaptation. That is not to say, however, that black holes could not also be such adaptations. Just as eyes and reduced skin pigmentation could both be BNSselected adaptations, life and black holes could both be CNSselected adaptations. But just as eyes are more likely to be an 
adaptation than reduced melanin, life is more likely to be an adaptation than black holes.

\section{Relationship of These Ideas to Other CNS and CNS-I Theories}

The main goal of this paper is to present an adaptationist revision of the CNS theory $[1,9,10]$ which proposes black holes as the most likely mechanism of universe replication. If we assume, as CNS theory does, that universes reproduce themselves, then everything we know about how selection works (at the biological level) implies that life is more likely than black holes to be an adaptation for enabling this reproduction. This adaptationist perspective does not necessarily imply, however, that some form of intelligent life was responsible for designing our own universe. We already know, from observing BNS, that a nonintentional, purely mechanistic selective process can be a masterful designer of functional complexity; no intelligence or foresight is required. From this perspective, the conditions of our universe that seem finely tuned to permit life to exist [16] could be the result of an entirely mechanistic selective process. To the extent that intelligent life could eventually evolve into a relatively effective means of universe replication, biofriendly universe designs should become relatively well represented in a multiverse. An alternative CNS-I view is that our universe was in fact designed by some form of intelligent life in a parental universe [14]. Although this proposition should certainly be considered, it seems less parsimonious than the mechanistic model: in suggesting that adaptations are produced by something more elaborate than a mechanistic selective process, it makes assumptions that go beyond our observations of how adaptations are generated at the biological level. Further, it does not address the issue of how fine-tuning would emerge in the first place: unless we assume an infinite regression of biofriendly universes being produced by intelligent life, then we must assume that biofriendliness was at some point generated by a nonintelligent process.

\section{Conclusion}

The main considerations discussed above are summarized in Figure 1. If our observation of BNS is a reliable guide to what we should expect from CNS, then there are two especially interesting implications. The first is that although both intelligent life and black holes are plausible candidates to be CNS-designed adaptations, the probability of being such an adaptation is higher for life than it is for black holes (or any other known object in the universe). The second is that CNS may be the primary cause of cosmological order, just as BNS is the primary cause of biological order. In other words, much or all order we observe in the universe may ultimately be the product or by-product of selection for replication at the biological and cosmological levels. Without this order, there would be no entropic process (because nothing would decay to a less-ordered state) and therefore no arrow of time [17]. In sum, the process of selection may be far more fundamental

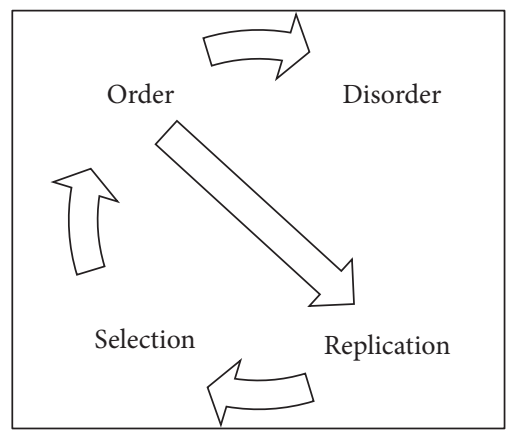

FIGURE 1: The selective and entropic processes as the primary causes of order and disorder. The linked concepts in the figure's upper half (order and disorder) represent the entropic process of ever-increasing disorder. The linked concepts in the lower half (replication and selection) represent processes of biological or cosmological natural selection: genetic or universe designs which outreplicate others achieve, via selection, greater representation in a population or multiverse. Selection leads to order because it produces the most orderly (and thus least entropic) known objects in the universe-adaptations-the function of which is to enable replication against the tide of the entropic process. Because adaptations must realize the complex goal of replication, they are characterized by improbable complex order, and such order is the hallmark of selection. The more improbably and complexly ordered something is (i.e., the lower its entropy), the more likely it is to be a biological or cosmological adaptation; intelligent life would thus be more likely than black holes (or anything else) to be a cosmological adaptation. Selection may be the main or only ultimate antientropic process in the universe/multiverse; that is, much or all order we observe may be the product or by-product of selection for replication at the biological and cosmological levels.

to explaining the nature of our universe than is generally supposed.

\section{Conflicts of Interest}

The author declares that there are no conflicts of interest regarding the publication of this paper.

\section{References}

[1] L. Smolin, The life of the cosmos, Oxford University Press, New York, NY, USA, 1997.

[2] J. Tooby, "Falling into place: entropy and the desperate ingenuity of life," in This Explains Everything, J. Brockman, Ed., Harper Perennial, 2013.

[3] C. R. Darwin, On the Origin of Species, John Murray, 1859.

[4] C. R. Darwin, The descent of man, and selection in relation to sex, John Murray, 1871.

[5] W. Hamilton, "The genetical evolution of social behaviour. I," Journal of Theoretical Biology, vol. 7, no. 1, pp. 1-16, 1964.

[6] G. C. Williams, Adaptation and natural selection: a critique of some current evolutionary thought, Princeton University Press, 1966.

[7] J. Tooby and L. Cosmides, "The psychological foundations of culture," in The Adapted Mind: Evolutionary Psychology and the 
Generation of Culture, J. Barkow, L. Cosmides, and J. Tooby, Eds., Oxford University Press, 1992.

[8] R. Dawkins, The Selfish Gene, Oxford University Press, 1976.

[9] L. Smolin, "Did the universe evolve?" Classical and Quantum Gravity, vol. 9, no. 1, pp. 173-191, 1992.

[10] A. Gardner and J. P. Conlon, "Cosmological natural selection and the purpose of the universe," Complexity, vol. 18, pp. 48-56, 2013.

[11] J. M. Smart, "Evo devo universe? A framework for speculations on cosmic culture," in Cosmos and Culture: Cultural Evolution in a Cosmic Context, S. J. Dick and M. L. Lupisella, Eds., pp. 201-295, Government Printing Office, NASA SP-2009-4802, Washington, DC, USA, 2009.

[12] C. Vidal, "The Beginning and the End: The Meaning of Life in a Cosmological Perspective," Springer, 2014.

[13] L. Crane, "Possible implications of the quantum theory of gravity: an introduction to the meduso-anthropic principle," Foundations of Science, vol. 15, pp. 369-373, 2010.

[14] E. R. Harrison, "The natural selection of universes containing intelligent life," Journal of the Royal Astronomical Society, vol. 36, pp. 193-203, 1995.

[15] J. N. Gardner, “The selfish biocosm," Complexity, vol. 5, pp. 3445, 2000.

[16] M. Rees, "Just six numbers: the deep forces that shape the universe," Basic Books, 2001.

[17] A. Eddington, "The Nature of the Physical World," MacMillan, 1928. 


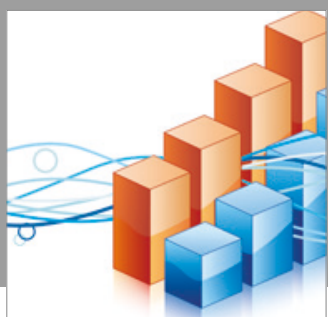

Advances in

Operations Research

vatersals

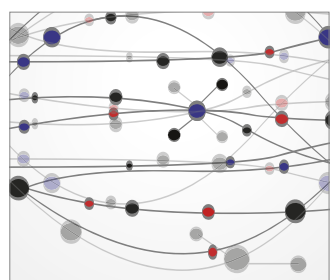

\section{The Scientific} World Journal
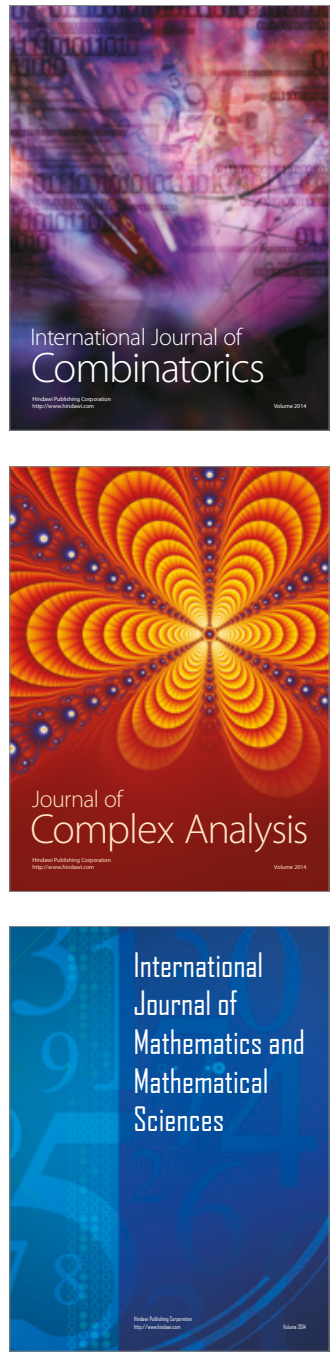
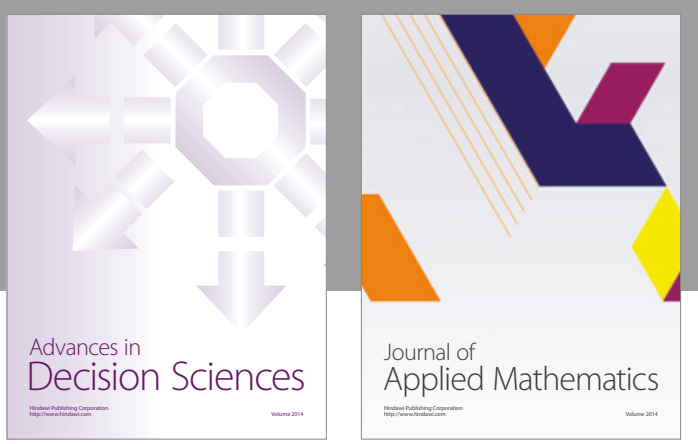

Algebra

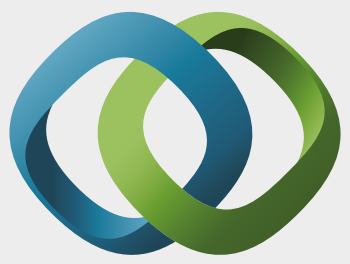

\section{Hindawi}

Submit your manuscripts at

https://www.hindawi.com
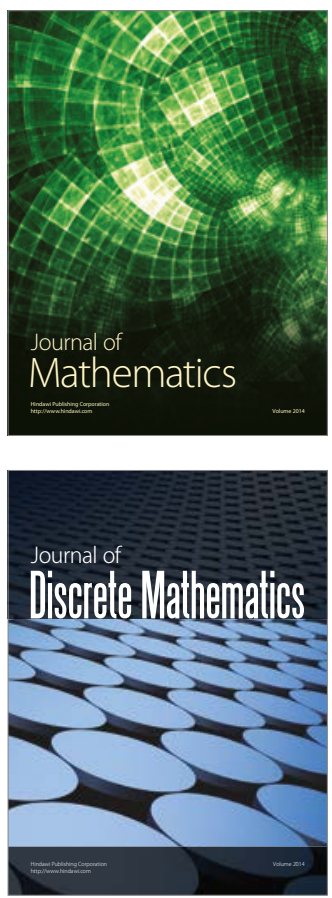

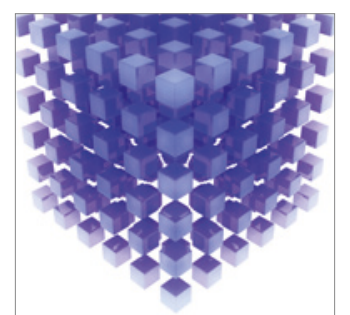

Mathematical Problems in Engineering
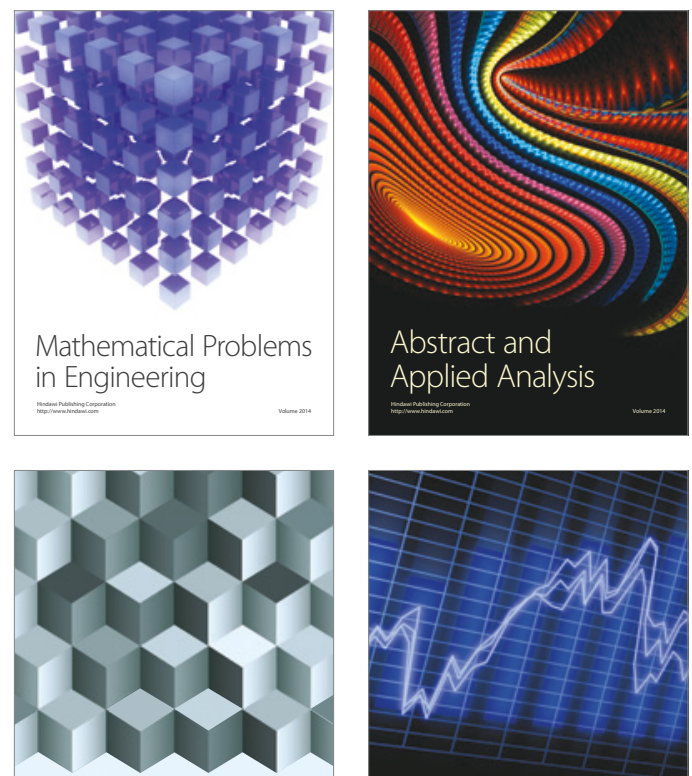

Journal of

Function Spaces

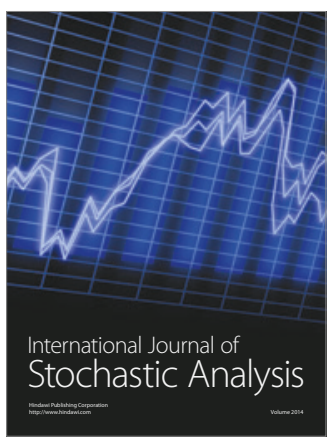

Probability and Statistics
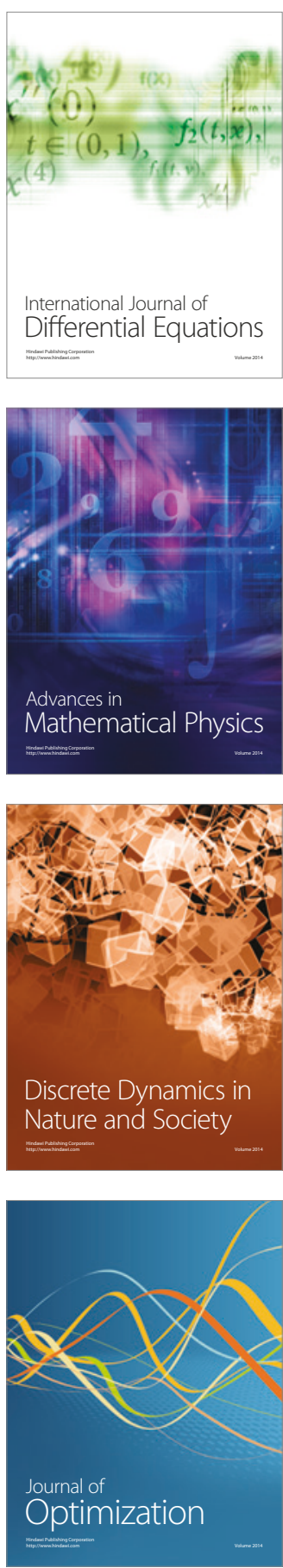